\title{
Imaging with DNA
}

\author{
DNA microscopy is an optics-free method for encoding physical images in DNA.
}

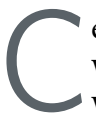
ell and tissue imaging provides a wealth of information on the inner workings of cells. The vast majority of methods for imaging cells involve microscopes with objective lenses for high-magnification views of specimens. However, there are also established alternative methods, such as imaging mass spectrometry, that involve taking spectra at individual points with known coordinates and combining them to form an image that reflects the chemical composition of the sample with spatial resolution.

Joshua Weinstein, Aviv Regev and Feng Zhang, of the Broad Institute, were inspired to create an alternative to both of these types of imaging. The researchers wanted to "see how genetic diversityover long stretches of hypervariable gene sequences-is distributed in space across cells of unknown identity," recalls Weinstein, who also notes that "these two forms of biological information are both entwined and inaccessible to researchers using current platforms." In addition, they wanted to avoid specialized equipment, such as custom light microscopes.

To meet these goals, the team developed DNA microscopy. According to the researchers, "DNA microscopy is premised on the notion that DNA can function as an imaging medium in a manner equivalent to light." In this method, DNA or RNA molecules within fixed cells are randomly tagged by DNA unique molecular identifiers, or UMIs. The DNA or RNA sequences are then amplified in situ so their positions can be 'broadcast' via the formation of a diffusion cloud. If a diffusion cloud overlaps with that of another molecule, the UMIs will concatenate, which encodes the proximity between the centers of the diffusion clouds. The reactions are then read out by deep sequencing, and a specialized algorithm is used to infer from the sequencing data the relative positions of all the original molecules, thus generating the $2 \mathrm{D}$ image of the sample.

As a first demonstration of the approach, the researchers asked whether they could

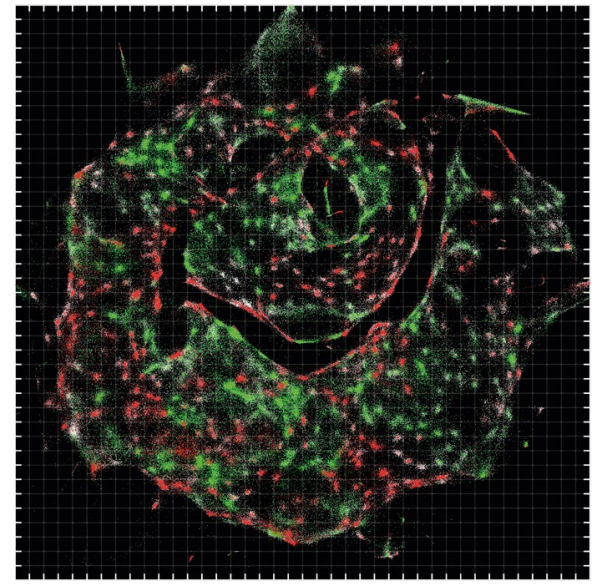

DNA microscopy image of a mixed population of cells expressing either green or red fluorescent protein. Reproduced from Weinstein et al. (2019), Elsevier

distinguish a mixed population of cells-one cell type expressing a red fluorescent protein, the other green-by DNA microscopy. They recovered images that were 'cell-like', and clearly saw regions that contained one fluorescent protein transcript or the other, while also seeing transcripts common to both cells in both regions-all without any prior knowledge of the organization of the cells. The quality of the performance, especially relative to fluorescence imaging of these cells, surprised even Weinstein, who notes, "The inferred image conformed not only in the relative positions of these cells but even in how individual axes scaled relative to each other." Indeed, the researchers further showed that the data enabled cell segmentation.

Despite these successes, getting the method up and running was not without hurdles. "I faced two main challenges in developing the technology," says Weinstein. "The first involved the problem of encoding an image of a specimen into DNA using a stand-alone chemical reaction." To solve this problem, they developed 'unique event identifiers', or UEIs, to assess the number of times the UMIs reacted with one another. "The second challenge involved the problem of decoding the physical image of UMItagged molecules computationally from the UEI data," notes Weinstein. Solving this problem required the development of specialized algorithms to handle millions of sequences that were robust to noise and downsampling.

Now that the technology is established, "the next question is, what are the areas of biology where DNA microscopy can make the greatest impact," says Weinstein, who notes that "elucidating hypervariability in cell populations in tumors, the immune system, the nervous system and the gut are all natural extensions of the technology." Weinstein also warns that "although DNA microscopy is very good at resolving very short distances between molecules, it is very bad at doing so over large distances, especially where there is empty space." Perhaps future extensions of the method can overcome this limitation.

DNA microscopy represents an exciting alternative to established methods for imaging cells and their unique nucleotidelevel variation simultaneously, and Weinstein shares that their team has already extended the method to imaging of whole transcriptomes, which has the added benefit of improving the resolution of the approach. Taken together, these results suggest that this approach will be useful for addressing a number of biological problems, especially those that are challenging to tackle with established methods. On a broader level, the method should encourage scientists to think beyond the microscope when they want to image biology.

Rita Strack

Published online: 30 July 2019

https://doi.org/10.1038/s41592-019-0524-6

Research papers

Weinstein, J. A. et al. DNA microscopy: optics-free spatio-genetic imaging by a stand-alone chemical reaction. Cell 178, 229-241 (2019). 